\title{
Laboratorio remoto utilizando dispositivos genéricos
}

MSc. Vázquez, Raimundo*; MSc. Spotorno, Rubén*; Ing. Liska, Diego*; MSc. Mariguetti, Jorge*

\section{Resumen}

En el presente trabajo se describe una experiencia de laboratorio remoto realizada en el área de Ingeniería Electromecánica, carrera que se dicta en la Facultad Regional Resistencia dependiente de la Universidad Tecnológica Nacional. El objetivo principal fue facilitar el proceso de aprendizaje del alumno y mejorar la práctica docente utilizando estrategias basadas en la indagación y la experimentación. Para tales fines se articuló aspectos formales y teóricos de termodinámica y fenómenos físicos observados en el mundo real.

Se partió del supuesto que el sujeto de aprendizaje que se inicia en la Ingeniería le resulta complejo relacionar leyes fundamentales de Termodinámica. A fin de poder facilitar el aprendizaje de conceptos relacionados con el aire húmedo, se partió de un laboratorio remoto desarrollado mediante la integración de dispositivos tecnológicos como por ejemplo: hardware genérico, tecnología móvil, conexión remota. Esto permitió cuantificar y predecir el comportamiento de variables termodinámicas. Esta modalidad de aprendizaje impactó tanto en el rol del docente como en el rol del alumno. El docente se convirtió en un guía encargado de

\footnotetext{
* GUDA-Grupo Universitario de Automatización | Universidad Tecnológica Nacional - Facultad Regional Resistencia French 414. 3500 Resistencia-Chaco. República Argentina | Tel: +54 362 4432928. / Fax: +54 3624432683 / e-mail: ray_vazquez_2005@hotmail.com
} 
sugerir posibles caminos y soluciones, por otro lado se diseñó material didáctico orientado a la experimentación mediante el uso de sensores digitales tales como: termohigrómetro, manómetros, barómetros, termómetros articulados con software libre que simplificó las tareas del docente. Se debió desarrollar habilidades relacionadas con el uso de ambientes de pruebas y laboratorios. El alumno debió desarrollar competencias que le permitan indagar, analizar, experimentar, contrastar y aplicar resultados, de manera tal de poder apropiarse significativamente de nuevos conocimientos.

Palabras clave: estrategias didácticas, laboratorio remoto, herramienta pedagógica

\section{Introducción}

El presente trabajo permite contribuir con el proceso de enseñanza aprendizaje de los fundamentos de las materias Termodinámica Técnica y Control Automático de Procesos, que se cursan en las carreras de Ingeniería Electromecánica y Química de la FRRe-UTN, utilizando como herramienta pedagógica un laboratorio remoto. Se busca generar recursos didácticos que permita al docente mejorar su práctica y facilitar en el aprendizaje, la apropiación de conceptos aplicados en el campo de la ingeniería, específicamente en el tema de laboratorio remoto utilizando como herramienta tecnológica un hardware genérico y software libre.

La enseñanza de conceptos de las materias mencionadas se encuadra dentro del área térmica y automatismo en la FRRe-UTN y tiene como principales desafíos formar los fundamentos que sustentarán en la introducción en el aprendizaje de nuevas tecnologías, y contribuirían a la transición que se da en el aprendiz en los primeros años en la universidad, en lo que respecta a la adquisición de competencias claves que requiere una carrera de grado. En este contexto, la enseñanza de conceptos termodinámicos y de automatización es posible abordar desde un enfoque clásico debido a la incorporación de esta tecnología específica en el área de la enseñanza aprendizaje, permitiendo incorporar el uso de estrategias de enseñanza desarrolladas en clases de teoría y en clases de práctica, estas últimas a su vez se basan principalmente en la resolución de guías de problemas y trabajos prácticos (CONFEDI, 2014). 


\section{Conceptos fundamentales}

\section{Laboratorios Remotos}

Existen muchas herramientas para simular un circuito electrónico, en ese sentido la idea del VISIR (Concari, 2012) permite implementar un circuito real remotamente, por lo cual se considera una gran iniciativa para la educación a distancia teniendo en cuenta lo que produce este tipo de educación: ahorro de coste, tiempo y menos riesgo, ya que el alumno no se encuentra en contacto físico con los instrumentos reales. La iniciativa de los laboratorios remotos nació en el instituto de tecnología de Massachusetts con su proyecto iLAB que pretende trabajar con circuitos electrónicos remotamente (Salah, 2015). Como se ha mostrado, el VISIR es capaz de sustituir las prácticas presenciales totalmente, por lo cual era una gran iniciativa del departamento de Ingeniería Eléctrica, Electrónica y Control Industrial de la UNED el aplicarlo en las asignaturas de Circuitos Eléctricos y Electrónicos. Se trata específicamente de la adquisición de una innovación educativa que permite facilitar el proceso de aprendizaje en áreas de la tecnología como por ejemplo electrónica y electricidad elemental.

\section{Entorno del Desarrollo Integrado}

Android Studio es el entorno de desarrollo integrado oficial para la plataforma Android.

Está disponible para las plataformas Microsoft Windows, macOS y GNU/Linux. Dicha herramienta permite implementar aplicaciones en dispositivos móviles (celulares o Tablet) en dos pasos. Se recurren a librerías de uso libre y código fuente abierta, que facilitan el manejo de: comunicación bluetooth, ubicación mediante tecnologías GPS, manejo de acelerómetros, visión artificial e inteligencia artificial (Teslyuk, 2015).

\section{Firebase de Google}

Firebase de Google es una plataforma en la nube para el desarrollo de aplicaciones web y móvil. Está disponible para distintas plataformas (iOS, Android y web), con lo que es más rápido trabajar en el desarrollo. Fue creada en 2011 y pasó a ser 
parte de Google en 2014, comenzando como una base de datos en tiempo real. Sin embargo, se añadieron más funciones que en parte, permitieron agrupar los SDK de productos de Google con distintos fines, facilitando su uso en fines de control automático, telemetría, estaciones remotas etc. En este trabajo de investigación, Firebase cumple la función esencial de hacer más sencilla la creación de aplicaciones móviles o APP y conexionado con dispositivos genéricos de bajos presupuestos.

\section{Hardware Genérico}

El Arduino Uno (Acakpovi, 2019) y (Rao, 2019) es un desarrollo electrónico de bajo presupuesto basado en un hardware flexible y fácil de usar, figura 1. Está pensado para artistas, diseñadores, como hobby y para cualquier interesado en crear objetos o entornos interactivos.

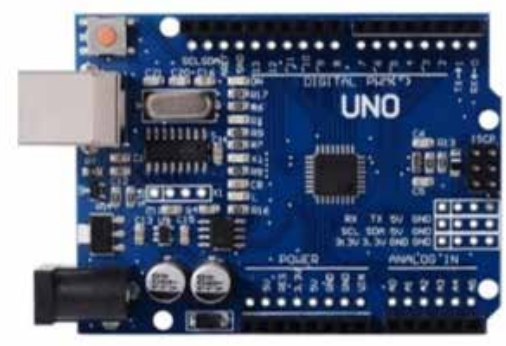

Figura 1: Arduino UNO

Puede realizar tareas de control y mediciones analógicas empleando un programa escrito de alto nivel (Yusuf, 2019). La innovación en el empleo de dispositivos genéricos Arduinos, es la incorporación de un software flexible y código fuente abierto facilitando la transferencia de información entre dispositivos mediante protocolos de comunicación SPI, I2C, RS232 y antenas inalámbricas. Se utiliza un transductor denominado sensor sumergible módulo DS18B20 como se muestra en la figura 2.

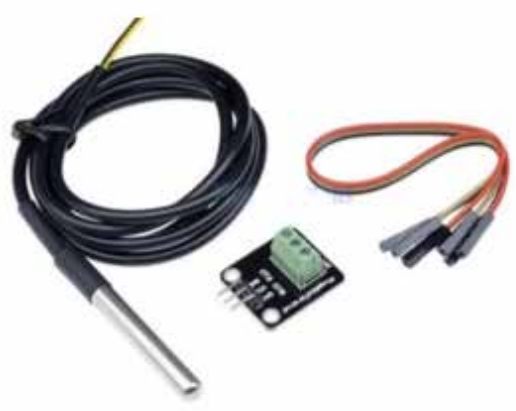

Figura 2: Sensor de temperatura 
En la figura 3 se visualiza un relé electrónico que facilita el manejo y control de potencia denominado relé de estado sólido.

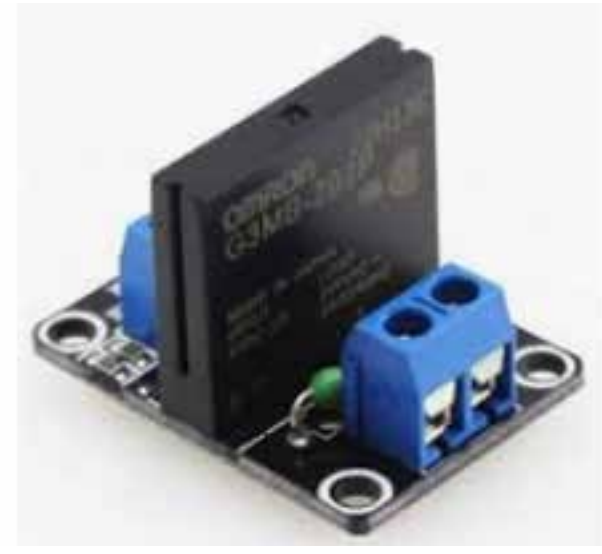

Figura 3: Módulo Relé de estado sólido

En la figura 4 se muestra un sensor encargado de almacenar en tiempo real las variables de estados termodinámicas de la experiencia de laboratorio.

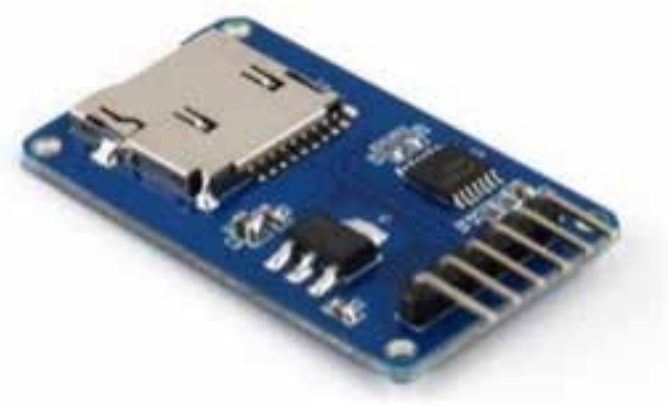

Figura 4: Sensor de Memoria

La figura 5 muestra un sensor de comunicación con tecnología bluetooth indispensable para vincular la información obtenida de los sensores y el hardware genérico.

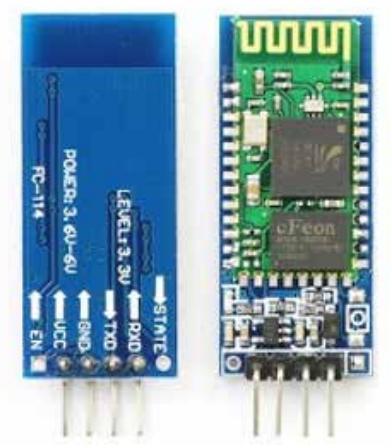

Figura 5: Módulo Bluetooth. 
El software encargado de configurar el funcionamiento del hardware genérico Arduino contempla la posibilidad de incluir librerías en el manejo y control de una variedad extensa de sensores y actuadores, facilitando la comunicación entre los diferentes sensores digitales. En la figura 6 se visualiza el sensor denominado DHT11 utilizado para adquirir datos de temperatura y humedad relativa.

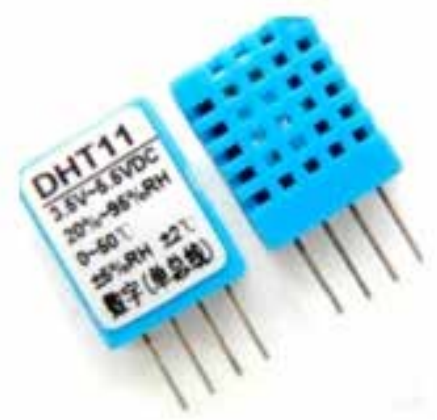

Figura 6: Módulo DHT11
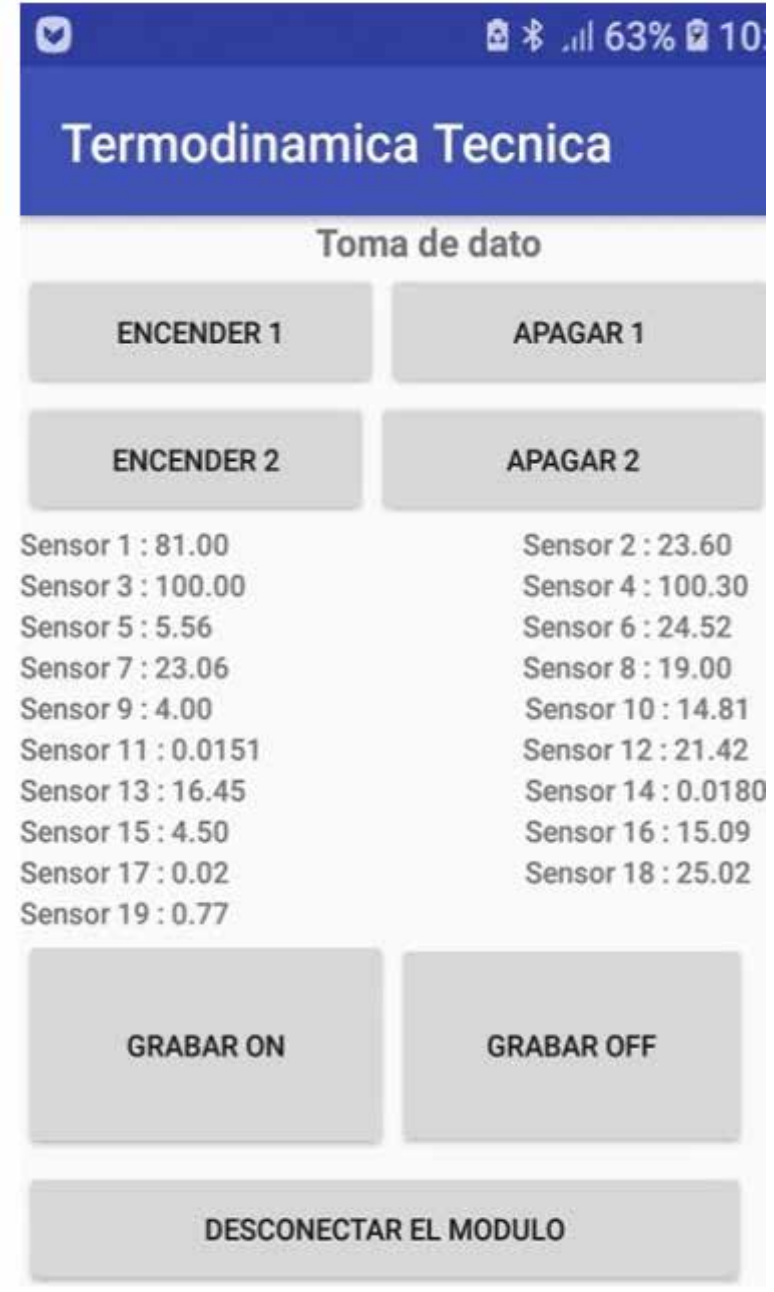

Figura 7: App desarrollada para el laboratorio remoto 


\section{Procedimiento}

Se utiliza un hardware genérico denominado Arduino UNO, software libre y herramientas de comunicación relacionada a la telemetría. La interconexión remota se facilita utilizando Firebase. De esta manera los valores obtenidos en el laboratorio remoto son visualizados en una página WEB de fácil configuración, requiriendo mínimos conocimientos de programación por parte de los docentes de la cátedra.

Los sensores utilizados son digitales y el fabricante brinda librerías de configuración permitiendo al docente implementar tareas de integración entre el hardware y envío de datos a la nube. El esquema simplificado de la experiencia se visualiza en la figura 8 .

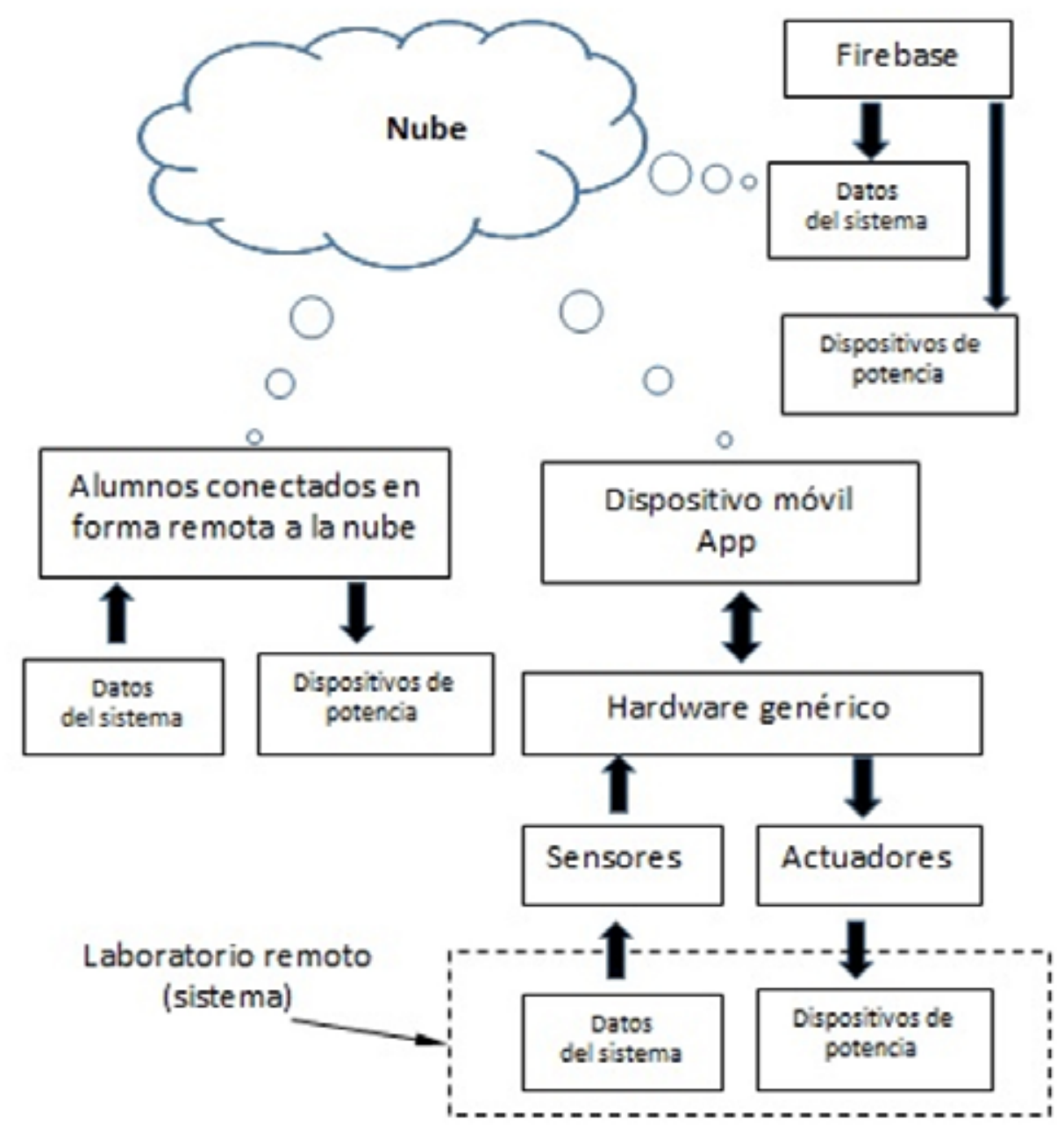

Figura 8: Esquema simplificado del laboratorio remoto 


\section{Desarrollo de la experiencia}

Se desarrolla un trabajo de laboratorio, donde se establece la necesidad de medir variables de estados tales como: temperatura, grado de saturación del aire húmedo, presión barométrica, presión manométrica. La guía de trabajos prácticos posee una breve introducción sobre la teoría del aire húmedo que el alumno deberá contrastar con los valores medidos en tiempo real en el laboratorio remoto. El docente en forma manual, puede modificar convenientemente los datos medidos por los sensores en el laboratorio remoto. De esta manera, el sistema evoluciona entre dos estados termodinámicos. Seguidamente los alumnos deberán interpretar el fenómeno físico y contrastar resultados utilizando las ecuaciones de la guía de laboratorio. Luego deberán realizar una predicción del estado final de equilibrio térmico utilizando las fórmulas de las transformaciones que actuaron en dichos procesos. El resultado de la predicción permite un criterio de control del relé encargado de manejar un dispositivo electromecánico dentro del laboratorio remoto. Es decir, si las variables termodinámicas alcanzan valores limites el alumno puede accionar desde su domicilio artefactos electromecánicos en forma remota.

\section{Resultados y discusión}

Del análisis de los resultados obtenidos de la guía de laboratorio entregados por los alumnos permitieron inferir las siguientes conclusiones:

a) Los aprendices mostraron interés, entusiasmo y compromiso con los nuevos conceptos incorporados durante los momentos de la práctica del laboratorio.

b) La experiencia impactó positivamente en los aprendices no solo al favorecer la apropiación de conceptos abstractos como por ejemplo, humedad, presión, temperatura etc. sino también al facilitar la construcción de un sentido de aplicación de un objeto de estudio, que en este caso fue manipulación y medidas de variables de termodinámicas.

c) La mayoría de los estudiantes por primera vez visualizaron claramente la representación de un fenómeno físico como por ejemplo: el proceso de humidificación del aire húmedo usando sensores digitales articulados con relé de estados sólidos.

d) La mayoría de los estudiantes resaltaron el potencial didáctico que posibilita: indagar, experimentar, observar, contrastar y predecir el estado final termodinámico con ecuaciones teóricas utilizando sensores digitales. 
e) La experiencia contribuyó a una mayor articulación e integración entre los docentes de teoría y práctica.

f) Se favoreció la reflexión colectiva por parte de los docentes acerca del impacto pedagógico del proceso y se contribuyó a repensar y resignificar la práctica docente.

\section{A continuación se plantean los principales interrogantes que fueron} surgiendo durante el proceso:

- ¿Se puede potenciar el autoaprendizaje del alumno utilizando este tipo de experiencias?

- ¿Cómo impacta en el rol docente la utilización de este tipo de laboratorio remoto?

- ¿Están los docentes preparados para incorporar este tipo de experiencias en otras áreas de la ingeniería?

- ¿Qué competencias claves favorece este tipo de experiencias para el alumno y docente?

- ¿Este tipo de prácticas puede complementarse con el aprendizaje basado en problemas?

- ¿Es fundamental la formación de un equipo sinérgico entre docentes, aprendices e ingenieros con experiencias en instrumentos y trabajo en laboratorios remotos?

- ¿Cómo podría mejorar la articulación entre teoría y práctica utilizando esta nueva herramienta tecnológica denominada hardware genérico?

- ¿Estamos preparados para encarar este tipo de experiencias en la FRReUTN?

- ¿Qué necesitamos para realizarlas, debido a que los recursos necesarios para su implementación son sustancialmente económicos?

- Este tipo de abordajes ¿̇se dan por la casualidad cuando los intereses de un grupo de docentes se encuentran y retroalimentan, debido a factores como la pandemia?

- ¿Es posible reproducirlas con otros temas y otros contextos? ¿Qué y cómo sistematizar?

- ¿Existen buenas prácticas que se puedan seguir y otras mejorar? 


\section{Conclusiones}

Se describió una experiencia realizada en el área de Ingeniería Electromecánica, que se dicta en la FRRe-UTN. El objetivo principal fue facilitar el proceso de aprendizaje del alumno y mejorar la práctica docente mediante la utilización de estrategias basadas en la indagación y experimentación. Para tales fines se articuló aspectos formales y teóricos y fenómenos físicos observados en el mundo real. Se partió del supuesto que al sujeto de aprendizaje que se inicia en la Ingeniería le resulta complejo relacionar leyes fundamentales de termodinámica. A fin de poder facilitar el aprendizaje de conceptos relacionados con el aire húmedo, se partió de un laboratorio desarrollado mediante la integración de dispositivos tecnológicos como por ejemplo hardware genéricos, tecnología móvil, conexión remota, software libre y sensores digitales que permitió cuantificar y predecir el comportamiento de variables termodinámicas. Esta modalidad de aprendizaje impactó tanto en el rol del docente como en el rol del alumno. El docente se convirtió en un guía o asesor encargado de sugerir posibles caminos y soluciones a problemas planteados.

Por otro lado, debe diseñar material didáctico fuertemente orientado a la experimentación mediante el uso de instrumentos, dispositivos y herramientas de análisis y observación. Se debió desarrollar habilidades relacionadas con el uso de ambientes de prueba y laboratorio. Además, el alumno debió desarrollar competencias que le permitan indagar, analizar, experimentar, contrastar y aplicar resultados, de manera tal de poder apropiarse significativamente de nuevos conocimientos relacionados con el tema de estudio. Esta modalidad le permitió al alumno el autoaprendizaje basado en la indagación y la comprobación de conceptos teóricos por medio de la experimentación.

\section{Referencias}

Amevi Acakpovi, Chiedozie Odazie, Issah Babatunde Majeed, George Eduful, Nana Yaw Asabere. Transformer Wireless Monitoring System Using Arduino/XBEE.

American Journal of Electrical Power and Energy Systems. ISSN: 2326-920o CONFEDI, Documentos de CONFEDI, Competencias en Ingeniería. Universidad 
Fasta 2014. ISBN: 978-987-1312-61-0. Pag-33.

Concari, S.; Kofman, H.; Marchisio, S. (2012) Laboratorios remotos y educación en Ingeniería. Entre retos y posibilidades. XVII Congreso Internacional de Tecnologías para la Educación y el Conocimiento: Tecnologías Emergentes. UNED. España.

Teslyuk Taras, Pavlo Denysyuk, Andriy Kernytskyy, Vasyl Teslyuk. Automated Control System for Arduino and Android Based Intelligent Greenhouse. MEMSTECH 2015, 2-6 September, 2015, Polyana-Svalyava (Zakarpattya), UKRAINE

Rao N, Narayana S, B.Ramu, D.Aruna , B. Deepika, B NEELIMA DEVI. SWITCH ON/OFF HOME APPLIANCES USING ARDUINO THROUGH VOICE COMMANDS. International Journal of Management, Technology AndEngineering . ISSN: $2249-7455$.

Salah, R.; Alves, G.; Abdulazeez, D.; Guerreiro, P.; Gustavsson, I. (2015) Why VISIR? Proliferative Activities and Collaborative Work of VISIR System. 7th International Conference on Education and New Learning Technologies (EDULEARN15) Proceedings, pp. 3824-3835.

Yusuf Abdullahi Badamasi Nigerian Turkish Nile University Abuja, Nigeria. The Working Principle of an Arduino. International Journal of Engineering Research \& Technology (IJERT). ISSN: 2278-0181. Vol. 4 Issue 01,January-2015.

\section{Abstract}

In the present work a remote laboratory experience carried out in the area of Electromechanical Engineering is described, a career that is dictated in the Regional Resistance Faculty of the National Technological University. The main objective was to facilitate the student's learning process and improve teaching practice using strategies based on inquiry and experimentation. For these purposes, formal and theoretical aspects of thermodynamics and physical phenomena observed in the real world were articulated. It was started from the assumption that the learning subject starting in Engineering finds it difficult to relate fundamental laws of Thermodynamics. In order to facilitate the learning of concepts related to humid air, we started from a remote labora- 
tory developed by integrating technological devices such as: generic hardware, mobile technology, remote connection. This allowed to quantify and predict the behavior of thermodynamic variables. This learning modality impacted both the teacher's role and the student's role. The teacher became a guide in charge of suggesting possible paths and solutions; on the other hand, didactic material oriented to experimentation was designed through the use of digital sensors such as: thermo-hygrometer, pressure gauges, barometers, articulated thermometers with free software that simplified the tasks of the teacher. Skills related to the use of test environments and laboratories had to be developed. The student had to develop skills that allow him to investigate, analyze, experiment, contrast and apply results, in such a way as to be able to significantly appropriate new knowledge.

Keywords: didactic strategies, remote laboratory, pedagogical tool 